\title{
OSCILLATION CRITERIA FOR CERTAIN SECOND ORDER NONLINEAR DIFFERENCE EQUATIONS
}

\author{
S.R. Grace and H.A. El-Morshedy
}

This paper is concerned with nonlinear difference equations of the form

$$
\Delta^{2} x_{n-1}+a_{n} f\left(x_{n}\right)=0, \quad n=1,2, \ldots
$$

where $\Delta$ is the forward difference operator defined by $\Delta u_{n-1}=u_{n}-u_{n-1}, \Delta^{2} u_{n-1}=$ $\Delta\left(\Delta u_{n-1}\right)$ and $\left\{a_{n}\right\}$ is a real sequence which is not assumed to be nonnegative. The function $f$ is such that $u f(u)>0$ for all $u \neq 0$ and $f(u)-f(v)=g(u, v)(u-v)$, for all $u, v \neq 0$, and for some nonnegative function $g$. Our results are not only new but also improve and generalise some recent oscillation criteria. Examples illustrating the importance of our main results are also given.

\section{INTRODUCTION}

In this paper we consider the second order difference equation

$$
\Delta^{2} x_{n-1}+a_{n} f\left(x_{n}\right)=0, \quad n=1,2, \ldots
$$

where $\Delta$ denotes the forward difference operator, that is, $\Delta u_{n-1}=u_{n}-u_{n-1}, \Delta^{2} u_{n-1}=$ $\Delta\left(\Delta u_{n-1}\right)$ and $\left\{a_{n}\right\}$ is a sequence of not necessarily nonnegative real numbers. The function $f: R \rightarrow R$ is continuous such that $u f(u)>0$ for $u \neq 0$. Throughout this work we consider $f(u)-f(v)=g(u, v)(u-v)$, for all $u, v \neq 0$, and for some nonnegative function $g$ if nothing else is assumed. The nonnegativity of $g$ means that $f$ is nondecreasing on $(0, \infty)$ and $(-\infty, 0)$. The function $g$ will be assumed to satisfy either one of the following conditions:

$$
g(u, v) \geqslant \lambda>0, \quad \text { for all } u, v \neq 0
$$

or

$$
\inf _{|u|,|v| \geqslant A} g(u, v)>0, \quad \text { for some } \quad A>0 .
$$

Received 4th January, 1999

The authors would like to thank Professor K. Gopalsamy for his helpful comments and suggestions on the original draft of this paper. H.A. El-Morshedy was on leave from the Department of Mathematics, Damietta Faculty of Science, New Damietta 34517, Egypt.

Copyright Clearance Centre, Inc. Serial-fee code: 0004-9729/99 \$A2.00+0.00. 
By the Mean Value Theorem, it is easy to see that if the function $f$ is differentiable on $R$ then (1.1) and (1.2) will be equivalent to

$$
f^{\prime}(u) \geqslant \lambda>0 \quad \text { for all } u \neq 0, \quad\left({ }^{\prime}=d / d u\right)
$$

and

$$
\inf _{|u| \geqslant A} f^{\prime}(u)>0 \text { for some } A>0
$$

respectively. We observe, also, that the class of all functions satisfying (1.2) contains not only those functions which satisfy (1.1) but also all superlinear functions; $f$ is called a superlinear function if

$$
\int_{\varepsilon}^{\infty} \frac{1}{f(x)}<\infty \text { and } \int_{-\varepsilon}^{-\infty} \frac{1}{f(x)}<\infty \text { for every } \varepsilon>0
$$

By a solution of equation $(E)$, we mean a real sequence $\left\{x_{n}\right\}$ which satisfies the equation $(E)$ for $n=0,1, \ldots$ A nontrivial solution $\left\{x_{n}\right\}$ of equation $(E)$ is said to be nonoscillatory if there exists $N \geqslant 0$ such that $x_{n+1} x_{n}>0$ for all $n \geqslant N$, otherwise it is called oscillatory. Equation $(E)$ is oscillatory if all of its solutions are oscillatory. Before proceeding further, we remark that the letters $i, J, k, n, N_{1}, N_{2}$ and $N$ below always denote positive integer variables.

A prototype of equation $(E)$ is the equation

$$
\Delta^{2} x_{n-1}+a_{n}\left|x_{n}\right|^{\nu} \operatorname{sgn} x_{n}=0, \quad \nu>0, \quad n=1,2, \ldots
$$

which is a discrete version of the well-known Emden-Fowler equation

$$
x^{\prime \prime}(t)+a(t)|x(t)|^{\nu} \operatorname{sgn} x(t)=0, \quad \nu>0, \quad t>0 .
$$

Hooker and Patula [5] obtained many results regarding the oscillatory properties of equation $\left(E_{1}\right)$ which correspond to certain discrete analogues of $\left(E_{2}\right)$. For $\nu>1$, that is, in the superlinear case, they have proved the following discrete version of the well-known Atkinson's criterion [1]:

TheOREM A. [5, Theorem 4.1] Suppose that $\nu>1$ and $a_{n} \geqslant 0$ eventually. Then equation $\left(E_{1}\right)$ is oscillatory if and only if

$$
\sum_{i=N}^{\infty} i a_{i}=\infty
$$

For equations of type $(E)$ when (1.1) is satisfied and $\left\{a_{n}\right\}$ is not assumed to be nonnegative, Thandapani et aliter $[13]$ proved the following result:

THEOREM B. [13, Theorem 3] In addition to (1.1), suppose that

$$
\sum_{i=N}^{\infty} a_{i} \text { exists, }
$$


and

$$
\liminf _{n \rightarrow \infty} A(n)>-\frac{1}{\lambda} \quad \text { where } \quad A(n)=\sum_{i=n}^{\infty} a_{i}
$$

If

$$
\sum_{i=N}^{\infty} \frac{A_{+}^{2}(i)}{1+\lambda A(i)}=\infty
$$

where $A_{+}(n)=\max \{A(n), 0\}$, then equation $(E)$ is oscillatory.

The previous result completes, partially, an earlier result by Szmanda [11] (see also $[13,15])$ given by the following:

ThEOREM C . [11] Assume that $f$ is a nondecreasing function on $R$. Then equation $(E)$ is oscillatory if

$$
\sum_{i=N}^{\infty} a_{i}=\infty .
$$

The following result for equation $(E)$ in the superlinear case has been established recently in [15]:

Theorem D. [15, Theorem 3.1] Assume that (1.3) and (1.5) are satisfied. If

$$
\lim _{n \rightarrow \infty} \sum_{k=N}^{n} \sum_{i=k+1}^{\infty} a_{i}=\infty,
$$

then equation $(E)$ is oscillatory.

Theorem $\mathrm{D}$ does not assume any restriction on the sign of $a_{n}$. But this theorem fails in a large class of equations because of condition (1.5).

It follows from the above introduction that the problem of finding oscillation criteria that avoid condition (1.5), as well as the nonnegativity assumption of $a_{n}$, is of particular interest. This problem was investigated in [3] for equation $(E)$ when $f(x)=x$; the aim of the present paper is to obtain some of those criteria, when either (1.1) or (1.2) is satisfied. Our main result of section 2 (that is, Theorem 2.1) is motivated by an open problem raised in [13] to impose a condition on $A(n)$ (defined by (1.6)) which is independent of $\lambda$, while the main result of section 3 (that is, Theorem 3.3) is motivated by Theorem A and Theorem B. The rest of our results improve and generalise some recent oscillation criteria in $[\mathbf{3}, \mathbf{1 3}]$. We also note that discrete analogues of some results for differential equations in $[2,4,6,7,9,10]$ are presented.

\section{Main Results}

ThEOREM 2.1. Assume that (1.1) holds and there exists a sequence $\left\{\phi_{n}\right\}$ satisfying

$$
\liminf _{n \rightarrow \infty} \sum_{i=N}^{n} a_{i} \geqslant \phi_{N}, \text { for every large } N
$$


and

$$
\sum^{\infty} \frac{\left(\phi_{i}^{+}\right)^{2}}{1+\lambda \phi_{i}^{+}}=\infty
$$

where $\phi_{n}^{+}=\max \left\{\phi_{n}, 0\right\}, \lambda$ is given by (1.1). Then equation $(E)$ is oscillatory.

Proof: Suppose that $\left\{x_{n}\right\}$ is a nonoscillatory solution of equation $(E)$; without loss of generality we assume that $x_{n}$ is eventually positive. Then, $x_{n}>0, n \geqslant N$ for some $N \geqslant 0$. Define $W_{n}$ as follows:

$$
W_{n}=\frac{\Delta x_{n-1}}{f\left(x_{n-1}\right)}, \quad n \geqslant N+1
$$

Using equation $(E)$,

$$
\Delta W_{n}=-a_{n}-\frac{\Delta x_{n-1} \Delta f\left(x_{n-1}\right)}{f\left(x_{n-1}\right) f\left(x_{n}\right)}, \quad n \geqslant N+1
$$

Consequently

$$
W_{n+1}-W_{N+1}=-\sum_{i=N+1}^{n} a_{i}-\sum_{i=N+1}^{n} \frac{\left(\Delta x_{i-1}\right)^{2} g\left(x_{i-1}, x_{i}\right)}{f\left(x_{i-1}\right) f\left(x_{i}\right)}
$$

But

$$
\frac{\left(\Delta x_{n-1}\right)^{2} g\left(x_{n-1}, x_{n}\right)}{f\left(x_{n-1}\right) f\left(x_{n}\right)} \geqslant 0, \text { for all } n \geqslant N+1
$$

implies that we have either

$$
\sum_{i=N+1}^{\infty} \frac{\left(\Delta x_{i-1}\right)^{2} g\left(x_{i-1}, x_{i}\right)}{f\left(x_{i-1}\right) f\left(x_{i}\right)}=\infty
$$

or

$$
\sum_{i=N+1}^{\infty} \frac{\left(\Delta x_{i-1}\right)^{2} g\left(x_{i-1}, x_{i}\right)}{f\left(x_{i-1}\right) f\left(x_{i}\right)}<\infty .
$$

Assume that (2.5) holds. Then (2.4) implies

$$
\lim _{n \rightarrow \infty} W_{n}=-\infty
$$

On the other hand, since $f(u)-f(v)=g(u, v)(u-v)$ for all $u, v \neq 0, f\left(x_{n}\right)-f\left(x_{n-1}\right)=$ $g\left(x_{n}, x_{n-1}\right) \Delta x_{n-1}$ for all $n \geqslant N+1$ which in view of (1.1) yields

$$
\begin{aligned}
W_{n} & =\frac{\Delta x_{n-1}}{f\left(x_{n-1}\right)}=\frac{f\left(x_{n}\right)}{f\left(x_{n-1}\right) g\left(x_{n}, x_{n-1}\right)}-\frac{1}{g\left(x_{n}, x_{n-1}\right)} \\
& >\frac{f\left(x_{n}\right)}{f\left(x_{n-1}\right) g\left(x_{n}, x_{n-1}\right)}-\frac{1}{\lambda} \\
& >-\frac{1}{\lambda}, \quad n \geqslant N+1 .
\end{aligned}
$$


Clearly, (2.8) contradicts (2.7). Therefore (2.5) can not hold and we consider now (2.6) which implies that

$$
\lim _{n \rightarrow \infty} \frac{\left(\Delta x_{n-1}\right)^{2} g\left(x_{n-1}, x_{n}\right)}{f\left(x_{n-1}\right) f\left(x_{n}\right)}=0
$$

or equivalently

$$
\lim _{n \rightarrow \infty} W_{n}^{2} \frac{g\left(x_{n-1}, x_{n}\right) f\left(x_{n-1}\right)}{f\left(x_{n}\right)}=0 .
$$

We have two possible cases for $W_{n}^{2}$; either $\limsup _{n \rightarrow \infty} W_{n}^{2}=M>0$ or $\lim _{n \rightarrow \infty} W_{n}^{2}=0$. Suppose the first case holds. Then there exists a subsequence $\left\{n_{k}\right\}$ such that $n_{k} \rightarrow \infty$ as $k \rightarrow \infty$ and $\lim _{k \rightarrow \infty} W_{n_{k}}^{2}=M$. Since $g\left(x_{n}, x_{n-1}\right) \geqslant \lambda>0$ for all $n \geqslant N+1$ (due to (1.1)); (2.9) yields

$$
\lim _{k \rightarrow \infty} \frac{f\left(x_{n_{k}-1}\right)}{f\left(x_{n_{k}}\right)}=0
$$

Then an intger $K$ can be chosen so large that

$$
f\left(x_{n_{k}-1}\right)<f\left(x_{n_{k}}\right) \text { for all } k \geqslant K .
$$

From the nondecreasing nature of $f$ on $(0, \infty) ;(2.10)$ implies that $x_{n_{k}}>x_{n_{k}-1}, k \geqslant K$ (that is, $\Delta x_{n_{k}-1}>0, k \geqslant K$ ) which in turn implies that $w_{n_{k}}>0$ for all $k \geqslant K$. Let $K_{1} \geqslant K$ be an integer such that $n_{k}>N+1$ for all $k \geqslant K_{1}$. Then replacing $n$ with $n_{k}$ in (2.4) we obtain

$$
W_{n_{k}}-W_{N+1}=-\sum_{i=N+1}^{n_{k}-1} a_{i}-\sum_{i=N+1}^{n_{k}-1} \frac{\left(\Delta x_{i-1}\right)^{2} g\left(x_{i-1}, x_{i}\right)}{f\left(x_{i-1}\right) f\left(x_{i}\right)}, \quad k \geqslant K_{1}
$$

or

$$
W_{N+1} \geqslant \sum_{i=N+1}^{n_{k}-1} a_{i}+\sum_{i=N+1}^{n_{k}-1} \frac{\left(\Delta x_{i-1}\right)^{2} g\left(x_{i-1}, x_{i}\right)}{f\left(x_{i-1}\right) f\left(x_{i}\right)}, \quad k \geqslant K_{1} .
$$

Taking the limit inferior of the above inequality as $k \rightarrow \infty$,

$$
W_{n} \geqslant \phi_{n}+\sum_{i=n}^{\infty} \frac{\left(\Delta x_{i-1}\right)^{2} g\left(x_{i-1}, x_{i}\right)}{f\left(x_{i-1}\right) f\left(x_{i}\right)}>\phi_{n}, \quad n \geqslant N+1 .
$$

On the other hand, if $W_{n}^{2} \rightarrow 0$ as $n \rightarrow \infty$, then $W_{n} \rightarrow 0$ as $n \rightarrow \infty$. So, by taking the limit superior of both sides of (2.4), we get that (2.11) holds in this case, too. Next, let a subsequence $\left\{i_{k}\right\}_{k=1}^{\infty}$ be defined by

$$
\left\{i_{k}\right\}_{k=1}^{\infty}=\left\{i \geqslant N+1: \phi_{i} \geqslant 0\right\} \text { and } i_{k} \rightarrow \infty \text { as } k \rightarrow \infty .
$$

Using (2.11),

$$
W_{i_{k}} \geqslant \phi_{i_{k}}, \quad i_{k} \geqslant N+1
$$

Also, the second inequality in (2.8) yields

$$
\frac{f\left(x_{i_{k}-1}\right) g\left(x_{i_{k}-1}, x_{i_{k}}\right)}{f\left(x_{i_{k}}\right)} \geqslant \frac{\lambda}{\lambda W_{i_{k}}+1}, \quad i_{k} \geqslant N+1
$$


From the above inequality, (2.12) and the fact that the function $H(x)=x^{2} /(\lambda x+1)$ is increasing on $[0, \infty)$, we have

$$
\begin{aligned}
\sum_{i=n}^{\infty} \frac{\left(\Delta x_{i-1}\right)^{2} g\left(x_{i-1}, x_{i}\right)}{f\left(x_{i-1}\right) f\left(x_{i}\right)} & \geqslant \sum_{k=1}^{\infty} \frac{\left(\Delta x_{i_{k}-1}\right)^{2} g\left(x_{i_{k}-1}, x_{i_{k}}\right)}{f\left(x_{i_{k}-1}\right) f\left(x_{i_{k}}\right)} \\
& \geqslant \lambda \sum_{k=1}^{\infty} \frac{\left(W_{i_{k}}\right)^{2}}{\lambda W_{i_{k}}+1}=\sum_{k=1}^{\infty} H\left(W_{i_{k}}\right) \\
& \geqslant \lambda \sum_{k=1}^{\infty} H\left(\phi_{i_{k}}\right)=\lambda \sum_{i=n}^{\infty} \frac{\left(\phi_{i}^{+}\right)^{2}}{\lambda \phi_{i}^{+}+1}
\end{aligned}
$$

In view of (2.6) and (2.13), we get

$$
\lambda \sum_{i=n}^{\infty} \frac{\left(\phi_{i}^{+}\right)^{2}}{\lambda \phi_{i}^{+}+1}<\infty, \quad n \geqslant N+1
$$

which contradicts (2.2). Thus equation $(E)$ can not have a nonoscillatory solution under our assumptions. This completes the prooof.

We note that if either

$$
\sum_{i=1}^{n}\left(\phi_{i}^{+}\right)^{2} \text { or } \sum_{i=1}^{n} \frac{\left(\phi_{i}^{+}\right)^{2}}{\lambda \phi_{i}^{+}+1}
$$

is convergent, then $\lim _{n \rightarrow \infty} \phi_{n}^{+}=0$. Hence, there exists a real number $M>0$ such that $\phi_{n}^{+} \leqslant M$ for all $n \geqslant 1$ and

$$
\left(\phi_{n}^{+}\right)^{2} \geqslant \frac{\left(\phi_{n}^{+}\right)^{2}}{\lambda \phi_{n}^{+}+1} \geqslant \frac{1}{\lambda M+1}\left(\phi_{n}^{+}\right)^{2} \text { for all } n \geqslant 1,
$$

which, by comparison, yields that the convergence of any one of the above two series implies that of the other. The following result is a consequence of Theorem 2.1 and the above analysis:

COROLlary 2.1. In addition to the conditions (1.1) and (2.1), assume that

$$
\sum^{\infty}\left(\phi_{i}^{+}\right)^{2}=\infty
$$

Then equation $(E)$ is oscillatory.

EXAmple 2.1. Consider the equation $(E)$ in which

$$
a_{n}=(-1)^{n} \frac{2 n^{2}+8 n+7}{(n+1)(n+2)}-\Delta \frac{1}{(n+1)^{1 / 2}}
$$

it is found that

$$
\sum_{i=N}^{n} a_{i}=(-1)^{n}+\frac{(-1)^{n}}{n+2}-\frac{1}{(n+2)^{1 / 2}}+(-1)^{N}+\frac{(-1)^{N}}{N+1}+\frac{1}{(N+1)^{1 / 2}}
$$


then

$$
\liminf _{n \rightarrow \infty} \sum_{i=N}^{n} a_{i}=-1+(-1)^{N}+\frac{(-1)^{N}}{N+1}+\frac{1}{(N+1)^{1 / 2}}, \quad N \geqslant 1 .
$$

Accordingly, there exists a sequence $\left\{\phi_{n}\right\}$ defined by

$$
\phi_{n}=-1+(-1)^{n}+\frac{(-1)^{n}}{n+1}+\frac{1}{(n+1)^{1 / 2}}, \quad n \geqslant 1
$$

and satisfying (2.1). Furthermore

$$
\phi_{n}^{+}= \begin{cases}\frac{1}{n+1}+\frac{1}{(n+1)^{1 / 2}} & \text { if } n \text { is an even integer } \\ 0 & \text { otherwise. }\end{cases}
$$

Therefore

$$
\sum_{i=1}^{\infty}\left(\phi_{i}^{+}\right)^{2} \geqslant \sum_{i=1}^{\infty} \frac{1}{i+1}=\infty
$$

In view of Corollary 2.1 , equation $(E)$ with the above $\left\{a_{n}\right\}$ is oscillatory. Moreover one can see that none of the results in $[5,11,12,13,14,15]$ can be used to examine the oscillation of $(E)$ in this case.

It follows from the proof of Theorem 2.1 that we can derive the next result:

COROLlary 2.2. Suppose that (1.1) is satisfied and furthermore

$$
\limsup _{n \rightarrow \infty} \sum^{\infty} a_{i}=\infty
$$

Then equation $(E)$ is oscillatory.

Proof: Proceeding as in the proof of Theorem 2.1, we obtain (2.4) and (2.8). But, (2.4) and (2.15) imply that $\liminf _{n \rightarrow \infty} W_{n}=-\infty$ which contradicts (2.8). This completes the proof.

EXAMPLE 2.2. Consider the following difference equation

$$
\Delta^{2} x_{n-1}+[1+\alpha n \sin (\pi(n-1) / 2)]\left(x_{n}+x_{n}^{3}\right)=0, \quad n=1,2, \ldots
$$

where $\alpha$ is any real number. We shall apply Corollary 2.2 to show that the above equation is oscillatory. Clearly, (1.1) is satisfied with $\lambda=1$. Furthermore

$$
\begin{aligned}
\sum_{i=N}^{n} a_{i}= & (n+1)\left(1-\frac{\alpha}{\sqrt{2}} \cos (\pi(2 n-1) / 4)\right)+\frac{\alpha}{2} \sin (\pi n / 2) \\
& -\frac{\alpha}{2} \sin (\pi(N-1) / 2)+N\left(-1+\frac{\alpha}{\sqrt{2}} \cos (\pi(2 N-3) / 4)\right)
\end{aligned}
$$

which leads to

$$
\limsup _{n \rightarrow \infty} \sum_{i=N}^{n} a_{i}=\infty
$$


Then (2.15) is satisfied and by Corollary 2.2 we conclude that all solutions of the above equation are oscillatory. One such solution is $\left\{x_{n}\right\}$ where $x_{2 n}=0$ and $x_{2 n+1}=(-1)^{n}$, $n=0,1, \ldots$.

REMARK 2.1. Corollary 2.2 extends and improves [3, Corollary 2.1] to more general equations. Also, Corollary 2.2 improves Theorem $\mathrm{C}$ when condition (1.1) is satisfied. Furthermore, since

$$
\limsup _{n \rightarrow \infty} \frac{1}{n} \sum_{k=N}^{n} \sum_{i=N}^{k} a_{i}=\infty
$$

implies (2.15) then Corollary 2.2 extends the discrete analogue of Wintner's criterion (2.16) to more general equations (see [3]). Finally, it enables us to obtain certain improvements of discrete analogues of known results for the differential equations, for example, [7, Corollary 1].

The last result in this section is concerned with the oscillation of equation $(E)$ when (2.2) fails, that is,

$$
\sum^{\infty} \frac{\left(\phi_{i}^{+}\right)^{2}}{1+\lambda \phi_{i}^{+}}<\infty
$$

In this case, one can define

$$
h_{0}(n)=\phi_{n}, \quad h_{1}(n)=\sum_{k=n}^{\infty} \frac{\left(h_{0}^{+}(k)\right)^{2}}{\lambda h_{0}^{+}(k)+1}
$$

and

$$
h_{i+1}(n)=\sum_{k=n}^{\infty} \frac{\left[\left(h_{0}(k)+\lambda h_{i}(k)\right)^{+}\right]^{2}}{\lambda\left(h_{0}(k)+\lambda h_{i}(k)\right)^{+}+1}, \quad i=1,2, \ldots
$$

where $h_{i}(n)$ will be assumed to obey the following condition:

There exists a positive integer $J$ such that

$$
h_{i}(n) \text { exists for } i=1,2, \ldots, J \text { and } \quad h_{J+1}(n)=\infty \text {. }
$$

THEOREM 2.2. Suppose that the conditions (1.1), (2.1) and (2.17) are satisfied. Then equation $(E)$ is oscillatory.

PROOF: As in the proof of Theorem 2.1, one can proceed to obtain (2.11) from which we get (2.13), that is,

$$
\sum_{i=n}^{\infty} \frac{\left(\Delta x_{i-1}\right)^{2} g\left(x_{i-1}, x_{i}\right)}{f\left(x_{i-1}\right) f\left(x_{i}\right)}>\lambda h_{1}(n) .
$$

By using (2.18) in (2.11), we obtain

$$
W_{n}>h_{0}(n)+\lambda h_{1}(n)
$$


From (2.19) together with similar arguments to those implying (2.13), one derives

$$
\sum_{i=n}^{\infty} \frac{\left(\Delta x_{i-1}\right)^{2} g\left(x_{i-1}, x_{i}\right)}{f\left(x_{i-1}\right) f\left(x_{i}\right)}>\lambda h_{2}(n) .
$$

Repeating this process, we get

$$
W_{n}>h_{0}(n)+\lambda h_{J}(n)
$$

hence

$$
\sum_{i=n}^{\infty} \frac{\left(\Delta x_{i-1}\right)^{2} g\left(x_{i-1}, x_{i}\right)}{f\left(x_{i-1}\right) f\left(x_{i}\right)}>\lambda h_{J+1}(n)=\infty,
$$

which contradicts (2.6). This completes the proof.

REMARK 2.2. Corollary 2.1 and Theorem 2.2 do not require that (1.5) or (1.6) is satisfied. Therefore, these results improve [13, Theorem 3 and 4], respectively, and answer the open problem of $[13, \mathrm{p} .206]$.

\section{Further Results}

Suppose that

$$
\liminf _{n \rightarrow \infty} \sum_{i=N}^{n} a_{i} \geqslant \phi_{N} \geqslant 0, \quad \text { for every large } N .
$$

We show that (3.1) enables us to know more information about the monotonicity of the nonoscillatory solutions of equation $(E)$. Strictly speaking, we have the following result which improves the discrete analogue of the well-known result of [2] for the differential equation

$$
y^{\prime \prime}(t)+a(t) f(y(t))=0, \quad t>0 .
$$

LEMma 3.1. Assume that $f$ is a nondecreasing function and (3.1) is satisfied. If $\left\{x_{n}\right\}$ is a nonoscillatory solution of equation $(E)$, then $x_{n} \Delta x_{n}>0$ eventually.

Proof: Suppose that $x_{n}$ is eventually positive. Then there exists an integer $N \geqslant 0$, such that $x_{n}>0$ for $n \geqslant N$. Define $W_{n}$ as in the proof of Theorem 2.1, we obtain (2.3) from which we get

$$
W_{n+1}-W_{N+1}=-\sum_{i=N+1}^{n} a_{i}-\sum_{i=N+1}^{n} \frac{\Delta x_{i-1} \Delta f\left(x_{i-1}\right)}{f\left(x_{i-1}\right) f\left(x_{i}\right)} .
$$

If the lemma is not true, then either $\Delta x_{n}<0$ eventually or $\Delta x_{n}$ oscillates. Assume that $\Delta x_{n}<0$ eventually; then in view of (3.1) one can choose $N_{1} \geqslant N$ such that $\sum_{i=N_{1}}^{n} a_{i} \geqslant 0$ and $\Delta x_{n}<0$ for all $n \geqslant N_{1}-1$. But by Abel's transformation $[8, \mathrm{p} .36]$ we have

$$
\sum_{i=N_{1}}^{n} a_{i} f\left(x_{i}\right)=f\left(x_{n+1}\right) \sum_{i=N_{1}}^{n} a_{i}-\sum_{i=N_{1}}^{n} \Delta f\left(x_{k}\right) \sum_{i=N_{1}}^{k} a_{i}, \quad \text { for all } n>N_{1}
$$


hence $\sum_{i=N_{1}}^{n} a_{i} f\left(x_{i}\right) \geqslant 0$ for all $n>N_{1}$. Then equation $(E)$ implies that

$$
\Delta x_{n} \leqslant \Delta x_{N_{1}-1}<0 \text { for all } n>N_{1}
$$

summing this inequality twice from $N_{\mathrm{l}}+1$ to $n$, then taking the limit as $n \rightarrow \infty$, we obtain that $x_{n} \rightarrow-\infty$ as $n \rightarrow \infty$ which contradicts the positivity assumption of $x_{n}$.

Suppose that $\Delta x_{n}$ oscillates. Then it is possible to choose $N_{2} \geqslant N$ such that $W_{N_{2}+1} \leqslant 0$. Hence, (3.2) implies that

$$
W_{n+1} \leqslant-\sum_{i=N_{2}+1}^{n} a_{i}-\sum_{i=N_{2}+1}^{n} \frac{\Delta x_{i-1} \Delta f\left(x_{i-1}\right)}{f\left(x_{i-1}\right) f\left(x_{i}\right)} .
$$

Taking the limit superior as $n \rightarrow \infty$ of the above inequality, we get $\limsup _{n \rightarrow \infty} W_{n+1}<0$ and since $W_{n}=\Delta x_{n-1} / f\left(x_{n-1}\right), \Delta x_{n}$ must be eventually negative which contradicts the oscillatory assumption of $\Delta x_{n}$. If $x_{n}$ is eventually negative, similar arguments imply a contradiction, too. This completes the proof.

EXAMPLE 3.1. Consider equation $\left(E_{1}\right)$ with

$$
a_{n}=\frac{2(n+1)^{\nu-1}}{(n+2) n^{\nu+1}}, \quad \nu>0, \quad n=1,2, \ldots
$$

Since $a_{n}>0$ for all $n=1,2, \ldots,(3.1)$ is satisfied. According to Lemma 3.1, any nonoscillatory solution of $\left(E_{1}\right)$, say $\left\{x_{n}\right\}$, with the above $\left\{a_{n}\right\}$ will satisfy $x_{n} \Delta x_{n}>0$ eventually. The reader can see easily that $x_{n}=n /(n+1)$ is one such solution.

THEOREM 3.1. Suppose that $f$ is a nondecreasing function on $R$ and (2.15), (3.1) are satisfied. Then equation $(E)$ is oscillatory.

Proof: Assume the contrary. Then there exists a nonoscillatory solution $\left\{x_{n}\right\}$ of equation $(E)$ which can be assumed to be eventually positive. By Lemma 3.1, $\Delta x_{n}$ is eventually positive. Now, proceeding as in the proof of Theorem 2.1, we obtain (2.4) which implies, in view of (2.15), that $\liminf _{n \rightarrow \infty} W_{n}=-\infty$ and hence $\Delta x_{n}$ can not be eventually positive. This contradiction completes the proof.

REMARK 3.1. Theorem 3.1 is a discrete analogue of [10, Theorem 2].

Now, if (3.1) holds and $\left\{x_{n}\right\}$ is a nonoscillatory solution of equation $(E)$, Lemma 3.1 leads to the existence of a positive real number $A$ and an integer $N>0$ such that $\left|x_{n}\right| \geqslant A$ for all $n \geqslant N$. So if (1.2) holds, one can find a real number $\mu>0$ such that

$$
g\left(x_{n-1}, x_{n}\right) \geqslant \mu \text { for all } n \geqslant N .
$$

Since in the proofs of all the rsults in Section 2 the left hand side of (1.1) is calculated along the nonoscillatory solutions of $(E)$, one can use (3.3) rather than (1.1) in these proofs provided that (1.2) and (3.1) are satisfied. In this case, new oscillation criteria can be obtained, from those of Section 2, that require $f$ to satisfy (1.2), which is weaker than (1.1). In particular, from Corollary 2.1 we get the following result: 
THEOREM 3.2. Suppose that the conditions (1.2) and (3.1) are satisfied. If

$$
\sum^{\infty}\left(\phi_{i}\right)^{2}=\infty
$$

then equation $(E)$ is oscillatory.

THEOREM 3.3. Suppose that $f$ is a nonodecreasing function on $R$ and (1.3), $(1.4)$ are satisfied. Then equation $(E)$ is oscillatory.

Proof: Let $\left\{x_{n}\right\}$ be a nonoscillatory solution of equation $(E)$. As usual, assume that $x_{n}$ is eventually positive, that is $x_{n}>0, n \geqslant N$ for some $N \geqslant 0$. Then $\Delta x_{n}$ is either eventually negative or eventually positive or oscillatory.

Suppose that $\Delta x_{n}$ is eventually negative. In view of (1.4), one can find $N_{1}>N$ such that

$$
\sum_{i=N_{1}}^{n} i a_{i} \geqslant 0, \text { and } \Delta x_{n-1}<0, \text { for all } n \geqslant N_{1} .
$$

By Abel's transformation, we obtain

$$
\sum_{i=N_{1}}^{n} i a_{i} f\left(x_{i}\right)=f\left(x_{n+1}\right) \sum_{i=N_{1}}^{n} i a_{i}-\sum_{i=N_{1}}^{n} \Delta f\left(x_{k}\right) \sum_{i=N_{1}}^{k} i a_{i} \geqslant 0
$$

then equation $(E)$ implies $\sum_{i=N_{1}}^{n} i \Delta^{2} x_{i-1} \leqslant 0$. But in view of [5, Lemma 2.2(b)], we have

$$
\sum_{i=N_{2}}^{n} i \Delta^{2} x_{i-1}=n \Delta x_{n}-\left(N_{1}-1\right) \Delta x_{N_{1}-1}-x_{n}+x_{N_{1}-1} .
$$

We let

$$
u_{n}=x_{n}-x_{N_{1}} \text { and } \quad \psi_{n}=-\sum_{i=N_{1}}^{n} i \Delta^{2} x_{i-1}-N_{1} \Delta x_{N_{1}-1}, \text { for all } n \geqslant N_{1},
$$

and get

$$
\Delta u_{n}-\frac{u_{n}}{n}+\frac{\psi_{n}}{n}=0, \quad n \geqslant N_{1}, u_{N_{1}}=0 .
$$

Then [14, Lemma 3.1] implies that

$$
u_{n}=-n \sum_{i=N_{1}}^{n-1} \frac{\psi_{i}}{i(i+1)}, \quad n=N_{1}+1, N_{1}+2 \ldots
$$

where $\psi_{n}>-N_{1} \Delta x_{N_{1}-1}>0$ for $n \geqslant N_{1}$. Hence

$$
u_{n}<-\left(-N_{1} \Delta x_{N_{1}-1}\right) n \sum_{i=N_{1}}^{n-1} \frac{1}{i(i+1)}=\left(N_{1} \Delta x_{N_{1}-1}\right) n\left(\frac{1}{N_{1}}-\frac{1}{n}\right) .
$$

As $n \rightarrow \infty$ the above inequality implies that $u_{n} \rightarrow-\infty$, which contradicts the positivity assumption of $x_{n}$. Thus, $\Delta x_{n}$ can not be eventually negative. Therefore, there exists a 
subsequence $\left\{n_{k}\right\}$ such that $n_{k} \rightarrow \infty$ as $k \rightarrow \infty$, and $\Delta x_{n_{k}} \geqslant 0, n_{k} \geqslant N_{2} \geqslant N$. Using Abel's transformation again, we get

$$
\sum_{i=N_{2}+1}^{n} \frac{i}{f\left(x_{i}\right)} \Delta^{2} x_{i-1}=\frac{n+1}{f\left(x_{n+1}\right)} \Delta x_{n}-\left(N_{2}+1\right) \frac{\Delta x_{N_{2}}}{f\left(x_{N_{2}+1}\right)}-\sum_{i=N_{2}+1}^{n} \Delta x_{i} \Delta \frac{i}{f\left(x_{i}\right)} .
$$

By equation $(E)$,

$$
\frac{n+1}{f\left(x_{n+1}\right)} \Delta x_{n}-\left(N_{2}+1\right) \frac{\Delta x_{N_{2}}}{f\left(x_{N_{2}+1}\right)}-\sum_{i=N_{2}+1}^{n} \Delta x_{i} \Delta \frac{i}{f\left(x_{i}\right)}+\sum_{i=N_{2}+1}^{n} i a_{i}=0
$$

or

$\frac{n+1}{f\left(x_{n+1}\right)} \Delta x_{n}-\left(N_{2}+1\right) \frac{\Delta x_{N_{2}}}{f\left(x_{N_{2}+1}\right)}+\sum_{i=N_{2}+1}^{n} \frac{i \Delta x_{i} \Delta f\left(x_{i}\right)}{f\left(x_{i}\right) f\left(x_{i+1}\right)}-\sum_{i=N_{2}+1}^{n} \frac{\Delta x_{i}}{f\left(x_{i+1}\right)}+\sum_{i=N_{2}+1}^{n} i a_{i}=0$.

Consider the above relation with $n=n_{k}$; we get

$\frac{n_{k}+1}{f\left(x_{n_{k}+1}\right)} \Delta x_{n_{k}}-\left(N_{2}+1\right) \frac{\Delta x_{N_{2}}}{f\left(x_{N_{2}+1}\right)}+\sum_{i=N_{2}+1}^{n_{k}} \frac{i \Delta x_{i} \Delta f\left(x_{i}\right)}{f\left(x_{i}\right) f\left(x_{i+1}\right)}-\sum_{i=N_{2}+1}^{n_{k}} \frac{\Delta x_{i}}{f\left(x_{i+1}\right)}+\sum_{i=N_{2}+1}^{n_{k}} i a_{i}=0$

and this implies

$$
-\left(N_{2}+1\right) \frac{\Delta x_{N_{2}}}{f\left(x_{N_{2}+1}\right)}+\sum_{i=N_{2}+1}^{n_{k}} i a_{i} \leqslant \sum_{i=N_{2}+1}^{n_{k}} \frac{\Delta x_{i}}{f\left(x_{i}\right)} .
$$

Define $u(t)=x_{n}+(t-n) \Delta x_{n}, n \leqslant t \leqslant n+1$ and $n \geqslant N_{2}+1$. It is easy to see that $u^{\prime}(t)=\Delta x_{n}\left(u^{\prime}(t)=d u(t) / d t\right)$. So if $\Delta x_{n} \geqslant 0$ then $x_{n} \leqslant u(t) \leqslant x_{n+1}$ for $n \leqslant t \leqslant n+1$ and the nondecreasing nature of the function $f$ yields

$$
f\left(x_{n}\right) \leqslant f(u(t)) \leqslant f\left(x_{n+1}\right), \quad n \leqslant t \leqslant n+1 \quad \text { and } n \geqslant N_{2}+1,
$$

which implies that

$$
\frac{\Delta x_{n}}{f\left(x_{n+1}\right)} \leqslant \frac{u^{\prime}(t)}{f(u(t))}, \quad n \leqslant t \leqslant n+1 .
$$

On the other hand, if $\Delta x_{n}<0 ; x_{n+1} \leqslant u(t) \leqslant x_{n}$ and $f\left(x_{n+1}\right) \leqslant f(u(t)) \leqslant f\left(x_{n}\right)$ for $n \leqslant t \leqslant n+1$. Therefore, (3.6) holds also for this case. Thus (3.6) is true for all $n \geqslant N_{2}+1$. Now, by (3.5) and (3.6), we have

$$
\begin{aligned}
-\left(N_{2}+1\right) \frac{\Delta x_{N_{2}}}{f\left(x_{N_{2}+1}\right)}+\sum_{i=N_{2}+1}^{n_{k}} i a_{i} & \leqslant \sum_{i=N_{2}+1}^{n_{k}} \int_{i}^{i+1} \frac{\Delta x_{i}}{f\left(x_{i+1}\right)} d t \\
& \leqslant \sum_{i=N_{2}+1}^{n_{k}} \int_{i}^{i+1} \frac{u^{\prime}(t)}{f(u(t))} d t \\
& =\int_{u\left(N_{2}+1\right)}^{u\left(n_{k}+1\right)} \frac{d u}{f(u)} \leqslant \int_{u\left(N_{2}+1\right)}^{\infty} \frac{d u}{f(u)}<\infty .
\end{aligned}
$$


Taking the limit as $k \rightarrow \infty$ in the above inequality, we obtain

$$
-\left(N_{2}+1\right) \frac{\Delta x_{N_{2}}}{f\left(x_{N_{2}+1}\right)}+\sum_{i=N_{2}+1}^{\infty} i a_{i} \leqslant \int_{u\left(N_{2}+1\right)}^{\infty} \frac{d u}{f(u)}<\infty
$$

which contradicts (1.4). Thus equation $(E)$ can not have a nonoscillatory solution provided that (1.3) and (1.4) are satisfied, which is our desired conclusion.

EXAMPLE 3.2. Consider the following equation

$$
\Delta^{2} x_{n-1}+\left(1+2(-1)^{n+1}\right)\left|x_{n}\right|^{\nu} \operatorname{sgn} x_{n}=0, \quad \nu>1, \quad n=1,2, \ldots
$$

In this case, the function $f(x)=|x|^{\nu} \operatorname{sgn} x$ satisfies (1.3). Furthermore, since $a_{n}=$ $1+2(-1)^{n+1}$, we find that

$$
\sum_{i=N}^{n} i a_{i}=n(n+1) / 2+(-1)^{n}(2 n+1) / 2-\left[N(N-1) / 2+(-1)^{N-1}(2 N-1) / 2\right] .
$$

Consequently, $\sum_{i=N}^{\infty} i a_{i}=\infty$ (that is, (1.4) is satisfied); by Theorem 3.3 we conclude that all solutions of the above equation are oscillatory. One such solution is $\left\{x_{n}\right\}$ where $x_{2 n}=0$, $x_{2 n+1}=(-1)^{n}(2 / 3)^{1 /(\nu-1)}$ for all $n=0,1, \ldots$

REMARK 3.2. Theorem 3.3 is sharp in the sense that when $a_{n} \geqslant 0$, eventually, (1.4) will become necessary and sufficient condition for the oscillation of equation $(E)$ as in Theorem A (see also [12, Theorem 1]). Since the sequence $\left\{a_{n}\right\}$ is not assumed to be eventually nonnegative, Theorem 3.3 improves the discrete analogue of Onose [9]. Finally, the continuous analogue of Theorem 3.3 can be found in $[4,6]$.

\section{REFERENCES}

[1] F.V. Atkinson, 'On second order nonlinear oscillations', Pacific J. Math. 5 (1955), 643-647.

[2] L. Erbe, 'Oscillation theorems for second order nonlinear differential equations', Proc. Amer. Math. Soc. 24 (1970), 811-814.

[3] S.R. Grace, A.A. Abadeer and H.A. El-Morshedy, 'On the oscillation of certain second order difference equations', Comm. Appl. Anal. 2 (1998), 447-456.

[4] J.R. Graef, S.M. Rankin and P.W. Spikes, 'Oscillation theorems for perturbed nonlinear differential equations', J. Math. Anal. Appl. 65 (1978), 375-390.

[5] J.W. Hooker and W.T. Patula, 'A second-order nonlinear difference equation: Oscillation and asymptotic behaviour', J. Math. Anal. Appl. 91 (1983), 9-29.

[6] I.T. Kiguradze, 'A note on the oscillation of solutions of equation $u^{\prime \prime}+a(t)|u|^{n} \operatorname{sgn} u=0$ ', (In Russian), Ćasopis Pèst. Mat. 92 (1967), 343-350.

[7] M.K. Kwong and J.S.W. Wong, 'Linearization of second order nonlinear oscillation theorems', Trans. Amer. Math. Soc. 279 (1983), 705-722.

[8] R.E. Mickens, Difference equations, theory and applications (Van Nostrand, Rheinhold, New York, 1990). 
(9) H. Onose, 'On oscillations of nonlinear second order equations', J. Math. Anal. Appl. 39 (1972), 122-124.

[10] H. Onose, 'Oscillation criteria for second order nonlinear differential equations', Proc, Amer. Math. Soc. 51 (1975), 67-73.

[11] B. Szmanda, 'Oscillation of solutions of second order difference equations', Portugal. Math. 37 (1978), 251-254.

[12] E. Thandapani, 'Oscillatory behaviour of solutions of second order nonlinear difference equations', J. Math. Phys. Sci. 25 (1991), 457-464.

[13] E. Thandapani, I. Györi and B.S. Lalli, 'An application of discrete inequality to second order nonlinear oscillation', J. Math. Anal. Appl. 186 (1994), 200-208.

[14] B.G. Zhang, 'Oscillation and asymptotic behaviour of second order difference equations', J. Math. Anal. Appl. 173 (1993), 58-68.

[15] B.G. Zhang and G.D. Chen, 'Oscillation of certain second order nonlinear difference equations', J. Math. Anal. Appl. 199 (1996), 827-841.

Department of Engineering Mathematics

Faculty of Engineering

Cairo University

Orman, Giza 1200

Egypt
Department of Mathematics and Statistics The Flinders University of South Australia GPO Box 2100

Adelaide SA 5001

Australia 